Vol.02/ No. 01

Pages: $1-12$

http://irojournals.com/jscp/

DOI: https://doi.org/10.36548/jscp.2020.1.001

\title{
Soft Computing Strategies for Optimized Route Selection in Wireless Sensor Network
}

\author{
Dr. Wang Haoxiang, \\ Director and lead executive faculty member, \\ GoPerception Laboratory, NY, USA \\ Email id: hw496@goperception.com.
}

Dr. S. Smys,

Professor, Department of CSE,

RVS Technical Campus,

Coimbatore, India.

Email id: smys375@gmail.com

\begin{abstract}
The routing in the wireless sensor networks has significant part in enhancing the functioning of the network as the improper routing methodologies and the routing deteriorates the energy of the sensor networks, affecting the lifespan of the network, leading to link failures and the connectivity problems. The necessity to improve the network performance is the main focus of the paper, so the paper presents the performance analysis of the evolutionary algorithms such as Genetic Algorithm, Ant lion optimization, Particle swarm optimization and Ant colony optimization and evaluates the routes obtained using the fuzzy Petri Net model, to find out the optimal route for the wireless sensor networks. The simulation through the python in terms of the some resource validates the optimal path in terms of energy, network life time and the packet delivery ratio.
\end{abstract}

Keywords: Soft Computing, Evolutionary Algorithms, Performance Analysis, Energy, Network Life Time and Packet Delivery Ratio

\section{Introduction}

The sensors were basically the electrochemical detectors for determining the physical extents. Initially these sensors were probably meant for the first thermostat rooms in the year 1933, and the sensors used in the early micro electrochemical systems where very large, costly and were mounted on separate chips packages. These sensors though large in size could not yield more. The latest advances in the MEMS as well as the ISSN: 2582-2640 (online) 
Journal of Soft Computing Paradigm (JSCP) (2020)

Vol.02/ No. 01

Pages: $1-12$

http://irojournals.com/jscp/

DOI: https://doi.org/10.36548/jscp.2020.1.001

integrated circuits paved way for the development of the tiny sensors that incorporated the actuators and few electronics alone. The technological improvements further have caused more advancement in the sensors allowing them to include on-board processor, memory and transceiver. Such sensors self organizes into a group constituting the network of wireless sensors. Every single sensor in the group is referred as the data point/ node in the wireless sensor network.

They nodes (sensors) were in built with the capabilities of the storage, processing and sensing and were fixed with the substructures with the networking facilities to transmit and receive an information. The WSN are widely used in diverse area of research and application as a solution to the innumerable problems. The Figure. 1 below shows the overview of the Wireless sensor network and the components in the sensors.

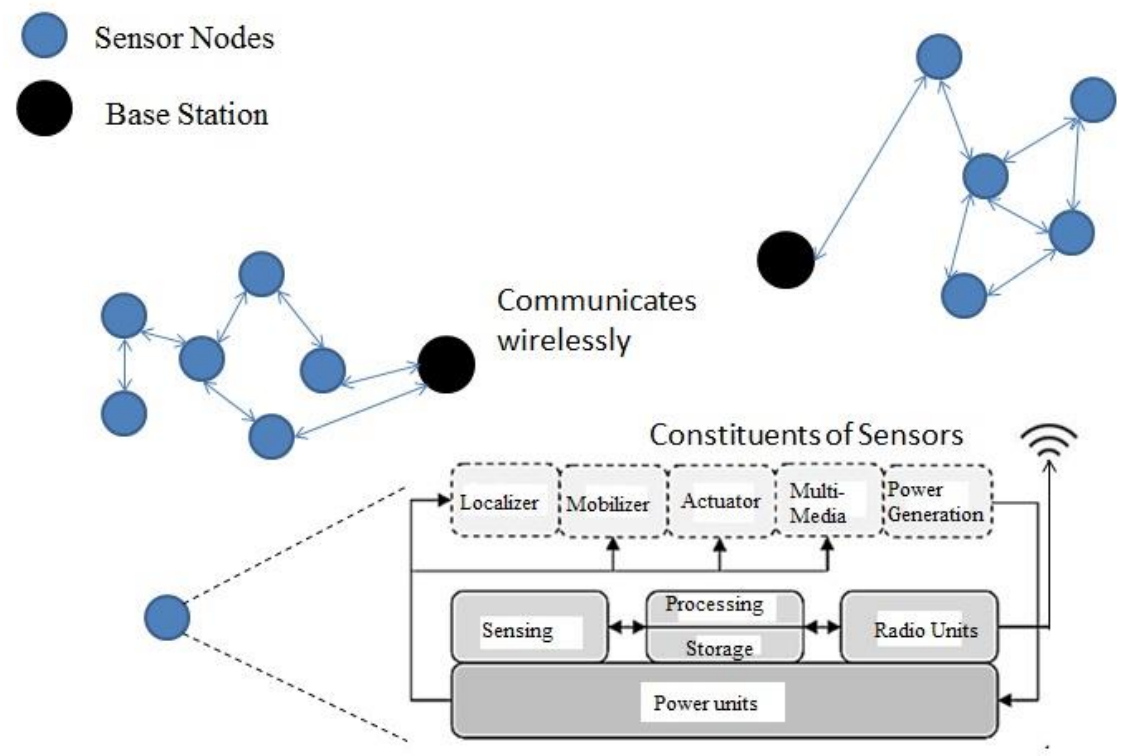

Figure.1 Wireless Sensor-Network Infrastructure

Though the self-organized network with the sensors has innumerable benefits as it could be deployed anywhere even in the places beyond human reach, providing low cost solutions for observing the environmental changes and remains as the very basic necessity of the today's internet of things, it suffers from few limitations as they are battery powered and do not poses a single topology due to their distributed

ISSN: 2582-2640 (online)

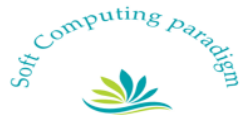


Journal of Soft Computing Paradigm (JSCP) (2020)

Vol.02/ No. 01

Pages: $1-12$

http://irojournals.com/jscp/

DOI: https://doi.org/10.36548/jscp.2020.1.001

nature. This nature of sensors often affects the lifetime of the sensors, resulting in the link failures, the improper data dissemination, blocking the data conveyance.

The functional enhancement of the wireless, networks framed using the sensors depends on the proper routing algorithm to elude the failures in the link by conserving the energy utilization and minimizing the delay in the transmission, numerous methods of routing either through reactive or the proactive manner were developed to reduce the energy usage and protect the network form link failures. From all the routing methods devised the evolutionary based routing that relied either on the evolution of the species or the foraging behavior of the species were found be highly preferred as they found optimal paths with enhanced energy optimization and security.

So paper observes the optimal routes obtained through the genetic algorithm, ALO, ACO and the PSO by analyzing the performance of the each method and evaluates all the routes employing the Fuzzy Petri Net, to find out the elite routing methodology that could be used to improve the performance of the network.

The remaining paper is organized with the related works that explains the various routing methodologies available in 2 . The proposed methodology for devising the routing and the applications of the evolutionary algorithms in it, and the evaluation structure of the fuzzy Petri Net (FP-N) in 3 , The performance analysis and the using Python and evaluation using FP-N in 4 and the Conclusion in 5

\section{Related Works}

Routing being an important and vital part in the wireless sensor networks, requires perfection in order to improve its performance without affecting the connection and the communications, multitudes of routing methods were framed for the wireless sensor network to optimize the energy in order to reduce the energy consumption prolonging the life time of the network, Salahuddin et al [1] provides the introduction to the "wireless sensor networks" explaining the infrastructure of the wireless sensor network, limitations and the routing methodologies, optimization of the energy so a prolong the life time of the network to make possible the seamless connections in the internet of things was put forth by the Raj, Jennifer S et al [2] utilizing the fuzzy logic and the CNN, and Smys, S., et al [3] propose the SDN based routing taking into consideration the mobility, energy consumption and the distance to develop an "A Stochastic Mobile Data Traffic Model For Vehicular Ad Hoc Networks." he also proposed in [4] the secure routing protocol for the wireless sensor networks in the big data applications using the cryptographic techniques.

ISSN: 2582-2640 (online) 
Journal of Soft Computing Paradigm (JSCP) (2020)

Vol.02/ No. 01

Pages: $1-12$

http://irojournals.com/jscp/

DOI: https://doi.org/10.36548/jscp.2020.1.001

Bhalaji, $\mathrm{N}$ et al [5] presented the routing for the vehicular network to improvise the packet delivery ratio and the throughput. Pandian, et al [6] proposes the utilization of evolutionary algorithms in the identifying the network for the heterogeneous wireless networks, Duraipandian, M et al [7] has utilized the "machine learning in the for the routing in the mobile adhoc networks", Pandian, M. Durai et al [8] has put forth an "Enhanced Network Performance And Mobility Management Of IOT Multi Networks" proposing an improved flow scheduling and mobility management. Sathesh, et al [9] proposes the "Optimized MultiObjective Routing for Wireless Communication with Load Balancing." Haoxiang, Wang, net al [10] provides the "QoS Enhanced Routing Protocols For Vehicular Network Using Soft Computing Technique." using the an lion based routing protocol.

Norouzi, et al [11] has proposed the "Genetic algorithm application in optimization of wireless sensor networks." Sun et al [12] has elaborated the "An improved routing algorithm based on ant colony optimization in wireless sensor networks." Kulkarni, et al [13] presents the survey on the particle swarm based routing optimization in the wireless sensor networks, all the above method consider any one or two metrics to reduce the energy consumption and prolong the network life.

The proposed method in the paper considers the energy conservation of the wireless sensor network by considering all the metrics such as the distance, residual energy, and trust of nodes, mobility, and the storage space to have a better performance optimization in the wireless sensor networks.

\section{Proposed Routing Protocol}

As the routing protocol plays a major role in improving the performance of the WSN, the proposed method tries to develop an efficient routing protocol considering all the metrics of that related in maintain the link stability and prolonging the life of the network. One of the key metrics affecting the lifetime of the network is the energy consumption $\left(E_{c o n}\right)$, as the sensors are powered by the battery and equipped with the limited battery power. The energy consumption is indirectly affected by many parameters such as the distance (Dist), where the distance could be the distance between the nodes $\left(\right.$ Dist $\left._{N}\right)$ in the network, the distance from node to the sink node ( ist $_{S N}$ ) or the distance form node to the target $\left(D_{i s t_{t g t}}\right.$ ), this is because the $D_{i s t_{N}}$ or the Dist $_{S N}$ or the Dist $t g t$ would cause delay in the conveyance increasing the utilization of the energy, the next metric indirectly affecting the $E_{c o n}$ would be the mobility of the nodes as the highly mobile sensor nodes if used in the network would leave the network at any time causing instability in the network connections paving way for reorganizing the network, resulting in energy consumption, and the other

ISSN: 2582-2640 (online)

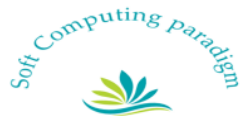


Journal of Soft Computing Paradigm (JSCP) (2020)

Vol.02/ No. 01

Pages: $1-12$

http://irojournals.com/jscp/

DOI: https://doi.org/10.36548/jscp.2020.1.001

factors are the storage space $\left(S_{S}\right)$ and the trust of the nodes $\left(t_{r}\right)$. The method put forth in the paper considers all the metrics mentioned above in selecting a node and to discover a path between the sources to the destination. The step below in the figure. 2 is the procedure followed in the proposed routing protocol.

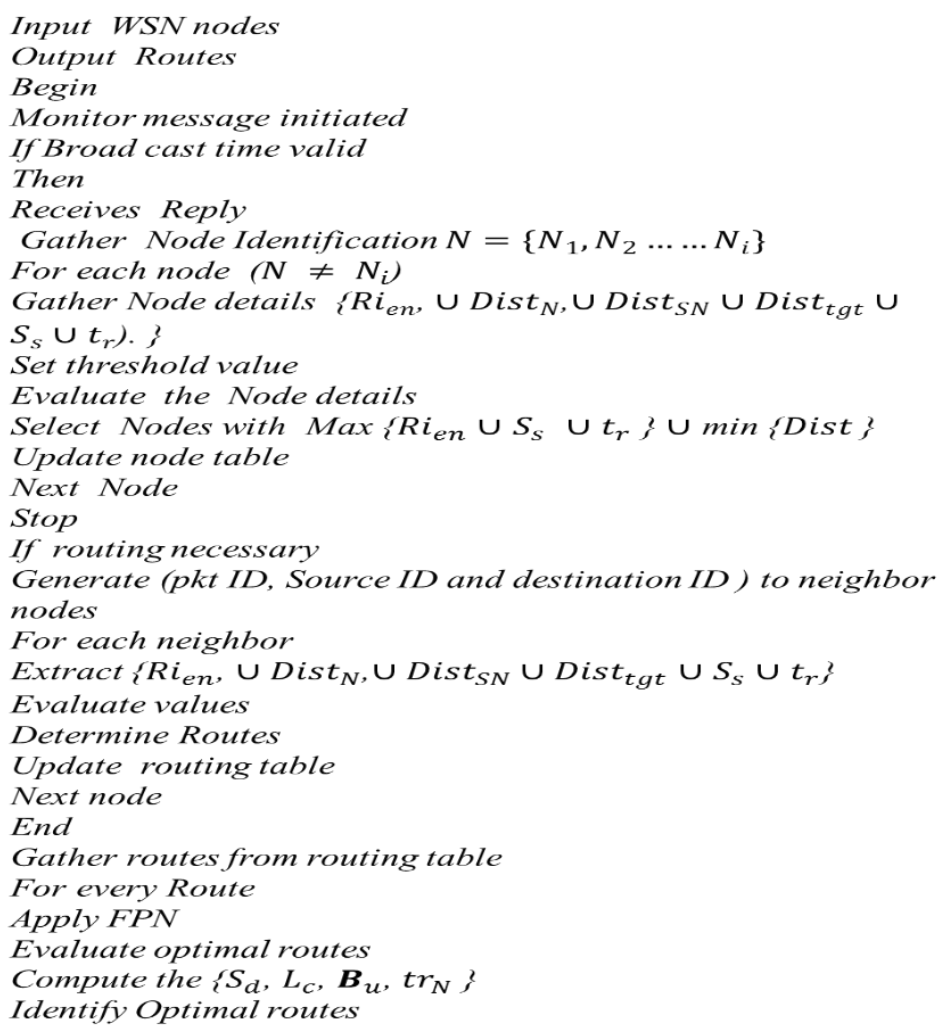

Figure.2 Algorithm

The each node $N=\left\{N_{1}, N_{2} \ldots \ldots N_{i}\right\}$ in the network is allotted with the identification number and every information to be sent is allotted with the packet identification $\left\{P k t_{1}, P k t_{2} \ldots \ldots . P k t_{n}\right\}$ each node holds completed transaction details and the particulars of the previous transactions/conveyance that has failed. The details of the completed transaction are gathered to ensure the trust of the nodes. The details of the of the nodes mobility, residual energy $\left(R i_{e n}\right), D i s t_{N}, D i s t_{S N}$, and the $D i s t_{t g t}$ are gathered to find out the optimality of the nodes, so as to engage them in the transmission. The selection of optimal nodes and routes is done by evaluating the nodes applying the evolutionary algorithms such as the genetic [11], particle swarm optimization [13], ACO [12] and the ALO [10] based on the $R i_{e n}$, Dist and the trust and the storage space and the mobility. The following equations are framed in this regard.

ISSN: 2582-2640 (online)

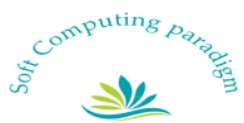


Journal of Soft Computing Paradigm (JSCP) (2020)

Vol.02/ No. 01

Pages: $1-12$

http://irojournals.com/jscp/

DOI: https://doi.org/10.36548/jscp.2020.1.001

$$
R i_{e n}=T O T_{e n}-\left(\left(\sum_{P k t=1}^{n} T r_{e n}+F w d_{e n}+P r_{e n}+R e_{e n}\right)+\sum\left(R o_{e n}+N s_{e n}\right)\right)
$$

The equation (1) gives the residual energy of the nodes which also known as the remaining energy of the nodes, the residual energy is calculate subtracting the TOT $_{\text {en }}$ (total energy available in the node), by the Average energy consumed in transmission $\left(T r_{e n}\right)$, in forwarding $\left(F w d_{e n}\right)$, in processing $\left(P r_{e n}\right)$ and in receiving the forwarded information $\left(R e_{e n}\right)$, along with the energy consumption in selecting nodes $\left(N s_{e n}\right)$ and the routes $\left(R o_{e n}\right)$. Nodes with the maximum $R i_{e n}$ are always opted in order to prolong the life time of the network as well as reduce the link failures.

The distance between the nodes are always expected to be low, in order to reduce the delay in the conveyance for e.g. for a source node $\left(S_{n}\right)$ the neighboring node $\left(N_{n}\right)$ that holds a very small with it is target node $\left(\operatorname{tg} t_{n}\right)$ selected as the next forwarding node. So the distance calculation to select the nodes with minimum distance is done using the following equation (2)

$$
\text { Dist }=\left\{\begin{array}{c}
\operatorname{dist}_{N_{n}}>\operatorname{dist}_{S_{n}} \\
\operatorname{dist}_{N_{n}}<\operatorname{dist}_{\text {tgt }_{n}}
\end{array}\right\}
$$

So a node is said to be optimal if has a minimum distance to its neighboring nodes as well as the target nodes. In the same way the node is expected to have a maximum storage space, trust and minimum mobility. Where the equation (3) provides the average trust of the nodes.

$$
A V G_{\text {trust }}=\left\{\begin{array}{l}
\delta * D_{\text {trust }}+\varphi * I D_{\text {trust }} \\
\delta * I D_{\text {trust }}+\varphi * D_{\text {trust }}
\end{array}\right\}
$$

Where the average trust of the nodes $\left(A V G_{\text {trust }}\right)$ is obtained determining the direct (D) and the indirect (ID) trust, $\delta$ and $\varphi$ are the success and the failure probabilities. Once the nodes are selected the route with the shortest distance $\left(S_{d}\right)$, less congestion $\left(L_{c}\right)$ and bandwidth utilization $\left(\boldsymbol{B}_{u}\right)$ and trusted nodes $\left(\operatorname{tr}_{N}\right)$ are selected applying the evolutionary algorithm, and evaluated applying the Fuzzy Petri Net to have the optimal route from the different set of routes selected applying different evolutionary algorithm. The figure. 3 below shows the Fuzzy Petri Net architecture in evaluating the routes to attain the optimal one.

ISSN: 2582-2640 (online) 
Journal of Soft Computing Paradigm (JSCP) (2020)

Vol.02/ No. 01

Pages: $1-12$

http://irojournals.com/jscp/

DOI: https://doi.org/10.36548/jscp.2020.1.001

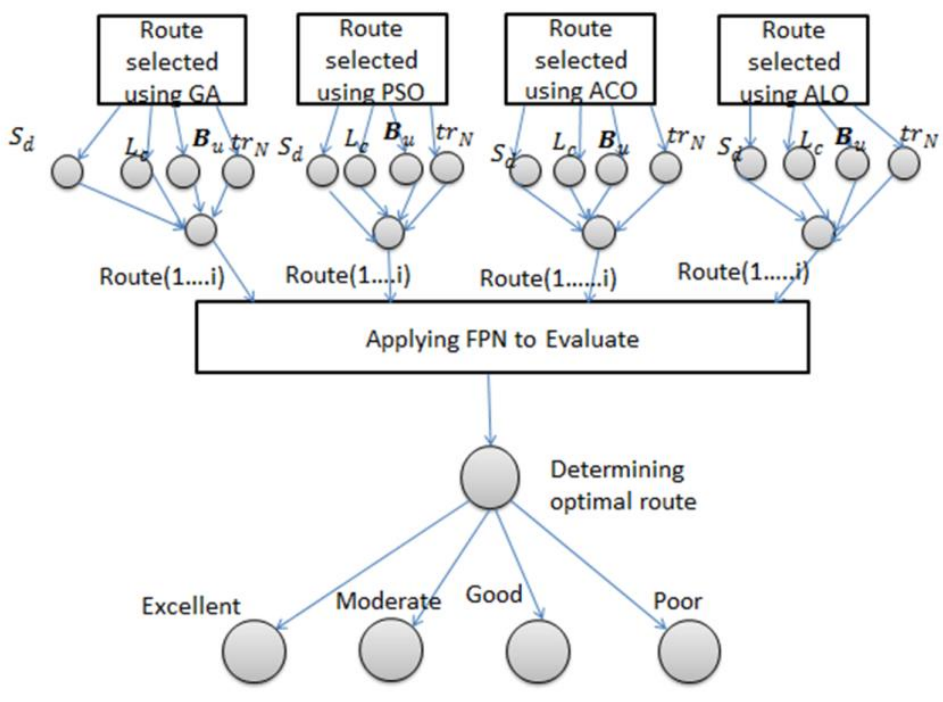

Figure.3 Evaluation Model of Fuzzy Petri Net (obtained from Chen, Q. et al [14] and modified)

The first step assigns priorities to the related elements to construct the un-weighted superiority matrix $\left(s u_{u w}\right)$ in order to build the decision index, in the next step the super matrix that is weighted $\left(s u_{w}\right)$ is achieved by multiplying the un-weighted superiority matrix $\left(s u_{u w}\right)$ and the weighted matrix $\left(W_{m}\right)$. Where thes $u_{w}=s u_{u w} * W_{m}$, the next step calculates the limit matrix, and constructs the evaluation model based on the Fuzzy Petri Net, the input values and the interior place token values are selected, and the threshold values to estimate the quality of the optimal routes are set and the finally the optimal routes are identified. The route evaluation model for the Fuzzy Petri Net was developed using the python and the optimal routes were estimated.

\section{Evaluation Results}

Each Route obtained from the Different EA methods were evaluated with the FPN developed using the python and the results were observed in the terms of the through put, energy consumption, network life time and packet delivery ratio for each method, for number of nodes varying form $500-1000$, in a simulation area of $2000 * 400$ sqm and a simulation time of 1000 seconds. The initial energy of the each node was estimated as 200 joules and the packet size of each packet was fixed to 2500 bytes.

ISSN: 2582-2640 (online)

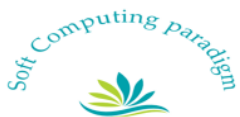


Journal of Soft Computing Paradigm (JSCP) (2020)

Vol.02/ No. 01

Pages: $1-12$

http://irojournals.com/jscp/

DOI: https://doi.org/10.36548/jscp.2020.1.001

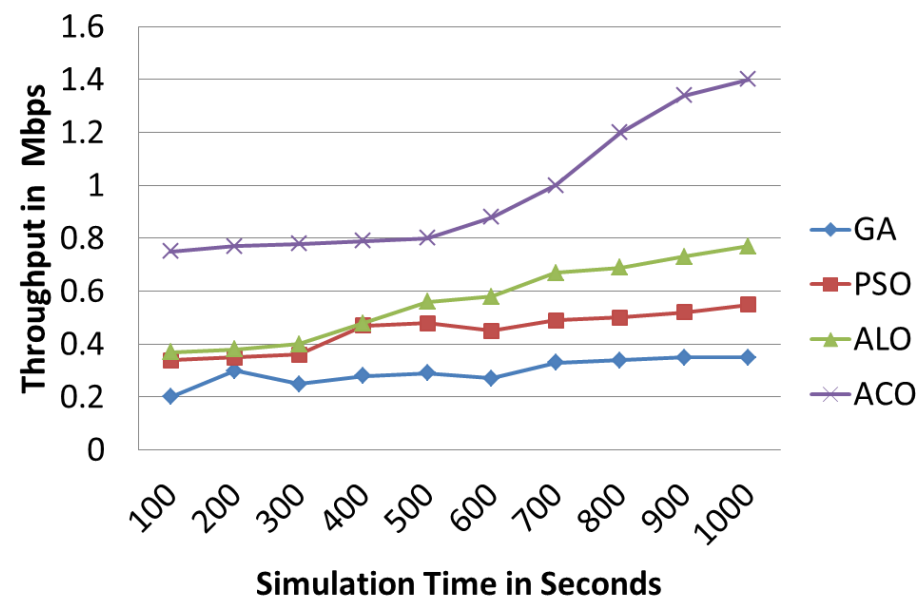

Figure.4 Throughput

The figure. 4 above shows the throughput achieved by the routes selected using the genetic algorithm, particle swarm optimization, ant colony optimization and the ant lion optimization. The evaluation through the FPN shows that the throughput achieved by the ant colony optimization is very much higher than the ALO, PSO and the Genetic algorithm as it finds out the shortest route, The shortest route identification reduces the delay in the data transmission as well as the minimizes the chances of security threat, achieving the maximum throughput.

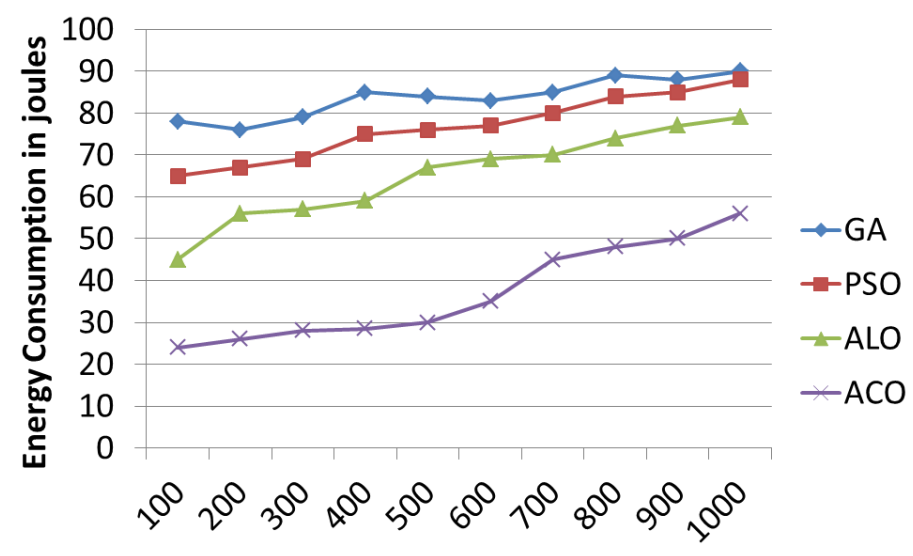

Simulation time in Seconds

ISSN: 2582-2640 (online) 
Journal of Soft Computing Paradigm (JSCP) (2020)

Vol.02/ No. 01

Pages: $1-12$

http://irojournals.com/jscp/

DOI: https://doi.org/10.36548/jscp.2020.1.001

Figure.5 Energy Consumption

From the results observed in the figure. 4 it is clear that the Ant colony optimization with the shorter distance reduces the delay and in turn reduces the energy consumption required in the transmission, the results in the fig. 5 show the energy consumption of the routes obtained using the genetic algorithm, particle swarm optimization, ant colony optimization and the ant lion optimization and shows that the energy consumption of the ACO was $15 \%$ lesser compared to the other algorithms.

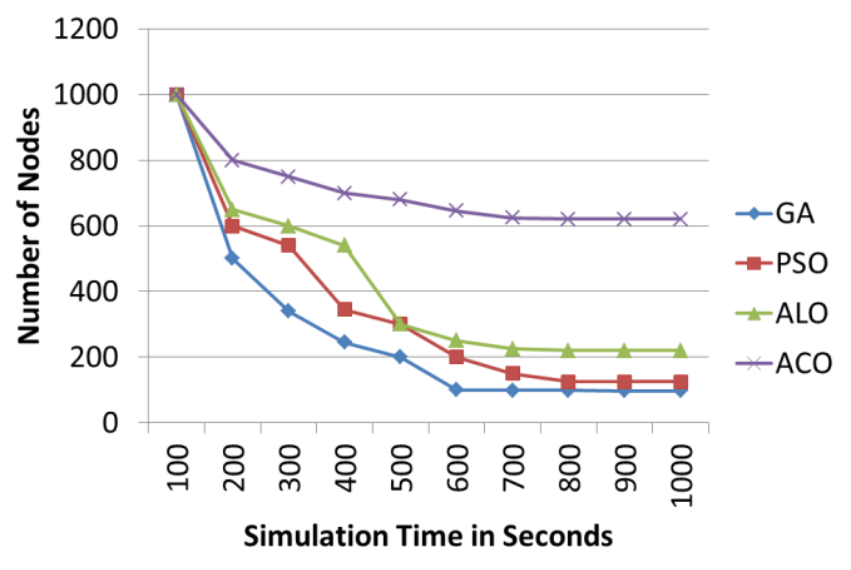

Figure.6 Network Life Time

As ACO is evinced to have a reduce power consumption the same algorithm shows an increased network life time (NLT) compared to the PSO, GA and the ALO, the ACO shows $10 \%$ higher NLT than the ALO and $20 \%$ higher NLT than the PSO and $35 \%$ higher NLT than the GA. The early convergence in the algorithms like GA and the PSO were reason for the local optimal route selection. Though ALO opted to have a global route selection the increased number of iterations to achieve a decision that is global optimal is more resulting in more power consumption and reduced network lifetime.

ISSN: 2582-2640 (online) 
Journal of Soft Computing Paradigm (JSCP) (2020)

Vol.02/ No. 01

Pages: 1-12

http://irojournals.com/jscp/

DOI: https://doi.org/10.36548/jscp.2020.1.001

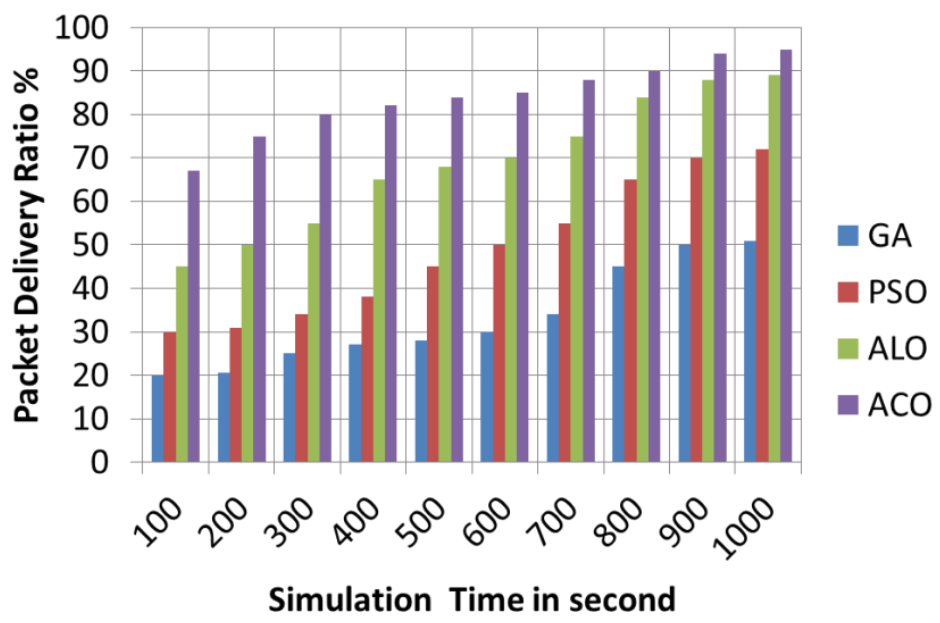

Fig.7 Packet Delivery Ratio

The Figure.7 showing the packet delivery ratio reveals that ACO has a better PDR compared to the other the PSO, GA and the ALO, as ACO shows $7 \%$ higher PDR than the ALO and $11 \%$ higher PDR than the PSO and $20 \%$ higher PDR than the GA. The table. 1 below shows the results observed for the each method.

\begin{tabular}{|c|c|c|c|c|c|c|c|c|c|c|c|c|c|c|c|c|}
\hline \multirow{2}{*}{$\begin{array}{l}\text { Number of } \\
\text { Nodes }\end{array}$} & \multicolumn{4}{|c|}{ GA } & \multicolumn{4}{|c|}{ PSO } & \multicolumn{4}{|c|}{ ALO } & \multicolumn{4}{|c|}{$\mathrm{ACO}$} \\
\hline & $\begin{array}{l}\text { TPT } \\
\text { (Mbp } \\
\text { s) }\end{array}$ & $\begin{array}{c}\mathrm{EC} \\
\text { (joule } \\
\text { s) }\end{array}$ & $\begin{array}{c}\text { PDR } \\
\%\end{array}$ & NLT & $\begin{array}{c}\text { TPT } \\
\text { (Mbp } \\
\text { s) }\end{array}$ & $\begin{array}{c}\mathrm{EC} \\
\text { (joule } \\
\text { s) }\end{array}$ & $\begin{array}{c}\text { PDR } \\
\%\end{array}$ & NLT & $\begin{array}{l}\text { TPT } \\
\text { (Mb } \\
\text { ps) }\end{array}$ & $\begin{array}{l}\mathrm{EC} \\
\text { (joul } \\
\text { es ) }\end{array}$ & $\begin{array}{c}\text { PDR } \\
\%\end{array}$ & NLT & $\begin{array}{c}\text { TPT } \\
\text { (Mbp } \\
\text { s) }\end{array}$ & $\begin{array}{c}\mathrm{EC} \\
\text { (joule } \\
\text { s) }\end{array}$ & $\begin{array}{c}\text { PDR } \\
\%\end{array}$ & NLT \\
\hline 100 & .2 & 78 & 30 & 1000 & .34 & 65 & 50 & 1000 & .37 & 45 & 70 & $\begin{array}{c}100 \\
0\end{array}$ & .75 & 24 & 85 & 1000 \\
\hline 200 & .3 & 76 & 34 & 500 & .35 & 67 & 55 & 600 & .38 & 56 & 75 & 650 & .77 & 26 & 88 & 800 \\
\hline 400 &. .28 & 85 & 45 & 245 & .48 & 75 & 65 & 345 & .48 & 59 & 84 & 540 & .79 & 28.5 & 90 & 700 \\
\hline 800 & .34 & 89 & 50 & 99 & .69 & 84 & 70 & 125 & 1.2 & 74 & 88 & 220 & 1.2 & 48 & 94 & 620 \\
\hline 1000 & .35 & 90 & 51 & 97 & .77 & 88 & 72 & 125 & 1.4 & 79 & 89 & 220 & 1.4 & 56 & 95 & 620 \\
\hline
\end{tabular}

Table.1 Comparison Table

ISSN: 2582-2640 (online) 
Journal of Soft Computing Paradigm (JSCP) (2020)

Vol.02/ No. 01

Pages: $1-12$

http://irojournals.com/jscp/

DOI: https://doi.org/10.36548/jscp.2020.1.001

The overall evaluation results shows that the routes discovered through the ant colony optimization is optimal compared to the other methods.

\section{Conclusion}

The paper has proposed a routing protocol to select the optimal nodes and the route to convey the information's, considering all the performance metrics such as $E_{c o n}$, Dist, residual energy, storage space, trust and mobility of the nodes has applied the algorithms GA, PSO ALO and ACO to evaluate the metrics of the nodes and select he optimal nodes to discover the routes with the minimum energy consumption, distance and maximum throughput and PDR. an evaluations model based on the fuzzy petri net was develop using python to evaluate the routes obtained using the different algorithms and categories the routes obtained using each algorithm as the excellent, moderate, good and bad. The Evaluation model rates the ACO to be the excellent one on the terms of throughput, energy consumption, network life time and PDR as it discovers the shortest route with higher, trust and limited congestion and bandwidth utilization, and rates the ALO to be good, the PSO to be the moderate and the GA as the worst due to its early convergence.

\section{References}

[1] Salahuddin, Mohammad Ali. "Introduction to wireless sensor networks." In Wireless sensor and mobile ad-hoc networks, pp. 3-32. Springer, New York, NY, 2015.

[2] Raj, Jennifer S. "QoS optimization of energy efficient routing in IoT wireless sensor networks." Journal of ISMAC 1, no. 01 (2019): 12-23.

[3] Smys, S., and Jennifer S. Raj. "A STOCHASTIC MOBILE DATA TRAFFIC MODEL FOR VEHICULAR AD HOC NETWORKS." Journal of Ubiquitous Computing and Communication Technologies (UCCT) 1, no. 01 (2019): 55-63.

[4] Smys, S. "ENERGY-AWARE SECURITY ROUTING PROTOCOL FOR WSN IN BIGDATA APPLICATIONS." Journal of ISMAC 1, no. 01 (2019): 38-55.

[5] Bhalaji, N. "PERFORMANCE EVALUATION OF FLYING WIRELESS NETWORK WITH VANET ROUTING PROTOCOL." Journal of ISMAC 1, no. 01 (2019): 56-71.

[6] Pandian, M. Durai. "ENHANCED NETWORK SELECTION AND HANDOVER SCHEMA FOR HETEROGENEOUS WIRELESS NETWORKS." Journal of ISMAC 1, no. 03 (2019): 160-171.

ISSN: 2582-2640 (online)

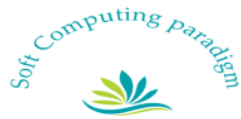


Journal of Soft Computing Paradigm (JSCP) (2020)

Vol.02/ No. 01

Pages: $1-12$

http://irojournals.com/jscp/

DOI: https://doi.org/10.36548/jscp.2020.1.001

[7] Duraipandian, M. (2019). PERFORMANCE EVALUATION OF ROUTING ALGORITHM FOR MANET BASED ON THE MACHINE LEARNING TECHNIQUES. Journal of trends in Computer Science and Smart technology (TCSST), 1(01), 25-38.

[8] Pandian, M. Durai. "ENHANCED NETWORK PERFORMANCE AND MOBILITY MANAGEMENT OF IOT MULTI NETWORKS." Journal of trends in Computer Science and Smart technology (TCSST) 1, no. 02 (2019): 95-105.

[9] Sathesh, A. "OPTIMIZED MULTI-OBJECTIVE ROUTING FOR WIRELESS COMMUNICATION WITH LOAD BALANCING." Journal of trends in Computer Science and Smart technology (TCSST) 1, no. 02 (2019): 106-120.

[10] Haoxiang, Wang, and S. Smys. "QOS ENHANCED ROUTING PROTOCOLS FOR VEHICULAR NETWORK USING SOFT COMPUTING TECHNIQUE." Journal of Soft Computing Paradigm (JSCP) 1, no. 02 (2019): 91-102.

[11] Norouzi, Ali, and A. Halim Zaim. "Genetic algorithm application in optimization of wireless sensor networks." The Scientific World Journal 2014 (2014).

[12] Sun, Yongjun, Wenxin Dong, and Yahuan Chen. "An improved routing algorithm based on ant colony optimization in wireless sensor networks." IEEE communications Letters 21, no. 6 (2017): 1317-1320.

[13] Kulkarni, Raghavendra V., and Ganesh Kumar Venayagamoorthy. "Particle swarm optimization in wireless-sensor networks: A brief survey." IEEE Transactions on Systems, Man, and Cybernetics, Part C (Applications and Reviews) 41, no. 2 (2010): 262-267.

[14] Chen, Q., Liao, S. L., \& Yi, S. P. (2015). A Fuzzy Petri nets/ANP Evaluation Model on Business Process Reengineering Implementation. In Proceedings of the 5th International Asia Conference on Industrial Engineering and Management Innovation (IEMI2014) (pp. 3-7). Atlantis Press, Paris.

\footnotetext{
ISSN: 2582-2640 (online)
} 